\title{
FLUOROSE DENTÁRIA É UM PROBLEMA EM SAÚDE PÚBLICA?
}

\author{
IS DENTAL FLUOROSIS \\ A PUBLIC HEALTH PROBLEM?
}

\author{
FÁBIO CARLOS KOZLOWSKI ${ }^{1}$ \\ VITOLDO ANTONIO KOZLOWSKI JÚNIOR² \\ 1 Mestrando em Odontologia, na área de Cariologia \\ na FOP/UNICAMP, Cirurgião-Dentista do Centro \\ Federal de Educação Tecnológica do Paraná - Uni- \\ dade de Ponta Grossa \\ 2 Professor do Departamento de Odontologia da \\ UEPG
}

\begin{abstract}
RESUMO
Apesar de ainda hoje a cárie dentária ser considerada no Brasil o principal problema de saúde bucal, o declínio em sua prevalência e severidade fazem com que a odontologia volte sua atenção para outros problemas da boca, como câncer, maloclusões e fluorose dentária. O aumento na prevalência da fluorose dentária verificado em nível mundial e também em várias regiões do país, nos faz questionar se ele caracteriza ou não um problema de saúde pública. Tentando responder este questionamento, os autores fazem uma discussão acerca da fluorose dentária e das características que determinam um problema em saúde pública.
\end{abstract}

Palavras-chave: fluorose dentária; saúde pública; flúor 


\section{Introdução}

Caracterizar e hierarquizar as patologias orais como sendo problemas de odontologia social e coletiva e conseqüentemente de saúde pública, envolve a necessidade de se conhecer uma multiplicidade de variáveis extra-odontológicas que condicionam a existência ou não dessas doenças, influenciando, inclusive, diretamente no ritmo e velocidade com que elas se expandem em uma determinada população. Um importante exemplo é quando consideramos a cárie dentária como sendo uma doença multifatorial que ocorre devido à interação de três fatores primários (essenciais): o hospedeiro (dentes e saliva), a microbiota e a dieta (KEYES, 1960), modulada pelo fator tempo, e cujo resultado é a lesão de cárie (NEWBRUN, 1978). Há, ainda, a influência de fatores socioeconômicos e comportamentais que podem contribuir para a sua instalação (FEJERSKOV e MANJI, 1990) e desenvolvimento, destruindo as estruturas dentais.

\section{Problema em Saúde Pública}

A Saúde Pública foi definida por McGavran, em 1956, como sendo a ciência responsável pelo diagnóstico e tratamento dos problemas de saúde das comunidades, e em uma visão mais atualizada e ampla, conceituada por Forattini, em 1996, como sendo a "atividade social que tem por precípuo objetivo promover e preservar a saúde da população, utilizando-se principalmente dos conhecimentos auferidos pelo estudo dos determinantes exógenos da doença ou agravo".

Tomando-se por referência esses conceitos, a odontologia social pode então ser definida como "a disciplina de saúde pública responsável pelo diagnóstico e tratamento dos problemas de saúde bucal da comunidade”, e desta forma, seus problemas caracterizados também como problemas de saúde pública, sendo dependentes diretamente do conceito entre o que é comum e o que é raro em determinada população ou região. (CHAVES,1986).

Segundo Sinai, em Chaves (1986), um problema de saúde é considerado como sendo problema de saúde pública a partir do momento em que preenche três condições essenciais:

1. Constitui causa comum de morbidade ou mortalidade; 
2. Existem métodos eficazes de prevenção e controle;

3. Tais métodos não estão sendo utilizados de modo adequado pela comunidade.

A Organização Mundial de Saúde - OMS, em 1954, classificou algumas doenças que constituem risco à saúde bucal: cárie, periodontopatias, maloclusões, lábio leporino, fendas palatais e câncer de boca. A essa relação, Striffler, em 1956, acrescentou ainda, fluorose dentária, seqüelas de traumatismos faciais, estigmas de sífilis congênita e doenças ocupacionais com manifestações bucais. No entanto, ao se discutir os problemas de odontologia social há necessidade do estabelecimento de critérios de modo a ordená-los de acordo com seu significado social, normalmente sendo utilizados, segundo Chaves (1986), os seguintes:

1. Número de pessoas atingidas;

2. Seriedade do dano causado;

3. Possibilidade de atuação eficiente

4. Custo per capita;

5. Grau de interesse da comunidade.

Desse modo, ao hierarquizar um certo problema, determinam-se as prioridades para sua futura erradicação. Tomando-se por base os princípios doutrinários do SUS (Sistema Único de Saúde) que regulamentam as ações de saúde no Brasil, ou seja, Universalização, Eqüidade e Integralidade, de modo especial, a Eqüidade, objetivando diminuir as desigualdades, investindo mais onde a necessidade e a carência são maiores, portando-se como um princípio de justiça social (CUNHA e CUNHA, in: CAMPOS et al., 1998), a priorização de determinadas doenças bucais torna-se, portanto, relevante. Contudo, priorizar não significa dar exclusividade e sim ênfase, pressupondo-se que o problema que ficou em segundo plano será contemplado e combatido com menor volume de recursos ou de tempo, porém sem ser esquecido ou abandonado (KRIGER, 1999).

Via de regra, o maior problema de ordem odontológica é a cárie dentária, pois contempla de modo irrefutável os critérios para estabelecimento de um problema de saúde pública (CHAVES, 1986). Há, no entanto, uma série de trabalhos demonstrando o decréscimo na prevalência de cárie dentária, tanto em nível mundial (AZCURRA et al., 1995; TRUIN et al., 1998), como em nível nacional (MOIMAZ et al., 1995; BASTING et al., 1997, FREIRE et al.; 1997; NARVAI et al., 1999).

Vários fatores podem estar contribuindo para que o perfil epidemiológico da cárie dentária apresente-se dessa maneira, tais como: 
modificações no padrão e quantidade de consumo de açúcar, maior acesso aos serviços odontológicos, melhoria nas condições de vida e ampliações das ações de promoção e educação em saúde bucal (SHEIHAM, 1984; DOWNER, in: BOWEN e TABAK, 1995; STEPHEN, in: BOWEN e TABAK, 1995) e de modo especial, o uso de fluoretos na busca do controle dessa doença (MURRAY, 1992). Entretanto, em função do aumento no consumo e uso inadequado de produtos contendo flúor, aumenta-se o risco de instalação da fluorose dentária, a qual se caracteriza como distúrbio específico da formação dos dentes causado por aumento crônico de depósitos de flúor durante o período de mineralização dentária (LESAN, 1987).

\section{Fluorose dentária}

De acordo com Besten (1994), fluorose dentária é uma hipoplasia do esmalte resultante da exposição à ingestão de flúor, sendo que seu grau de manifestação depende da dose de flúor ingerida, do tempo, da duração da exposição e da resposta individual de cada pessoa, considerando que em função dessas variáveis, doses similares de exposição ao flúor podem levar a diferentes níveis de manifestação clínica.

A fluorose apresenta distribuição simétrica entre os dentes homólogos (LARSEN et al., 1986; FEJERSKOV et al., 1990), sendo os dentes mais afetados os pré-molares, incisivos superiores e caninos (MOLLER, 1982), enquanto que na dentição decídua as características clínicas são similares às encontradas na dentição permanente, porém, com um padrão de severidade menor (THYLSTRUP, 1978). O período crítico com relação à exposição ao flúor em níveis acima dos terapêuticos passíveis de provocar sua manifestação no primeiro molar permanente, incisivos e pré-molares, é o compreendido entre o nascimento e os 11, 12 e 24 meses, (ISHI e SUCKLING, 1991).

Relatos na literatura, demonstram que, paralelamente à redução da incidência de cárie dentária, ocorreu um aumento das manifestações clínicas da fluorose dentária em áreas fluoretadas e não fluoretadas, em nível mundial (HEIFETZ et al., 1988; AKPATA et al., 1997; BARDSEN et al., 1999) e também em nível nacional (MARCELINO et al., 1999; TAVARES e BASTOS, 1999).

Esse aumento tem ocorrido principalmente nas categorias muito leve

PUBLICATIO UEPG - Biological and Health Sciences, 6 (1): 75-87, 2000. 
e leve (SEGRETO et al., 1984; SZPUNAR e BURT, 1987; BRUNELLE e CARLOS, 1990). Provavelmente isso tem ocorrido especialmente pela maior exposição a cremes dentais contendo flúor, em face da ingestão de parte deste dentifrício por crianças de baixa idade durante sua higienização bucal (STOOKEY, 1994; WANG et al., 1997; ARMONIA et al., 1999), até porque crianças acima de 8 anos estão livres de serem acometidas por fluorose, qualquer que seja o teor de flúor existente na água (BUENDIA e ZAINA, 1997).

Outros fatores concorrendo para o aumento da fluorose dentária seriam o uso de suplementos de flúor por indivíduos em áreas fluoretadas (KUMAR et al., 1989), consumo inadequado de suplementos de flúor na dieta (LEVY e ROZIER, 1987; LEVY, 1994) e uso de bochechos fluoretados em crianças menores de 6 anos de idade (CARLOS in: WEY, 1985).

A fluorose dentária pode ser mensurada por meio de vários índices, sendo os mais utilizados os índices de Dean (1942), T-F (THYLSTRUP e FEJERSKOV, 1978) e o TSIF (HOROWITZ et al., 1984). Pereira e Moreira (1999), que analisaram esses três índices de fluorose dentária ao examinarem 461 escolares entre 12 e 14 anos de idade, em três cidades do estado de São Paulo com concentrações diferentes de flúor (Iracemápolis $<0,3$ ppmF, Piracicaba 0,7 ppmF e Cesário Lange 1,4 ppmF), encontraram prevalências semelhantes de crianças afetadas pela fluorose, usando-se qualquer um dos índices, confirmando a possibilidade da comparação de levantamentos epidemiológicos independentemente do índice utilizado.

Osuji et al. (1988) conduziram estudo em East York, Ontario (fluoretada), em escolares de 8, 9 e 10 anos de idade, utilizando o índice TF, para mensurar a prevalência de fluorose, encontrando 13\% da amostra com graus variando de 1 a 4, não considerando desta forma, a fluorose dentária naquele momento, problema em saúde pública.

Ripa (1991), em análise crítica a respeito do uso de métodos de aplicação tópica de flúor, em um período demarcado pelo decréscimo da cárie dentária e aumento da prevalência da fluorose dentária em escolares americanos, recomenda o uso destes, de modo a influenciar ainda mais a redução da prevalência da cárie dentária, mas de modo racional e coerente, evitando-se assim o aumento da fluorose em termos de severidade, considerando que a fluorose em graus leves é esteticamente aceitável e insignificante, não se caracterizando assim, em problema de saúde pública.

Riordan (1993) afirma que a fluorose é o preço da prevenção, sendo que neste estudo, vinte e oito crianças com vários graus de fluorose 
mensurada pelo índice TF, variando de grau 0 a grau 3, permitiram o exame de seus dentes por 110 observadores em boas condições de visualização. Os observadores eram estudantes universitários, pais, funcionários públicos e dentistas, e quando perguntados acerca do aspecto agradável dos dentes, ocorreu concordância tanto entre os dentistas quanto entre os leigos, quando a condição dentária era de fluorose zero, mas à medida em que o escore de fluorose aumentou, essa concordância diminuiu.

Clark et al. (1993) determinaram a prevalência de fluorose dentária utilizando o índice TSIF em 1131 crianças em duas áreas, fluoretada e não fluoretada, em British Columbia, e em adição, os pais e as próprias crianças foram questionadas sobre o aspecto estético dos seus dentes. Dos examinados, $60 \%$ dos voluntários apresentaram fluorose em pelo menos duas superfícies, sendo que $8 \%$ tinham escores variando de 2 a 6 e $52 \%$ foram classificados na categoria 1 . Em relação à preocupação estética, a resposta fornecida pelos pais sugeriu haver pouca preocupação quando seus filhos encontravam-se no escore 1 , aumentando gradativamente à medida em que aumentava o escore.

Ellwood e O’Mullane (1995) determinaram a importância estética de diferentes tipos de defeitos do esmalte em voluntários provenientes de áreas com diferentes teores de flúor na água de abastecimento (0,1, 0,7 e $0,9 \mathrm{ppmF}$ ), questionando-os primeiramente a respeito da aparência de seus dentes e confrontando posteriormente suas respostas com fotografias. Em um segundo momento, dentistas e examinadores leigos foram levados a selecionar indivíduos que tivessem algum defeito. Em concordância com o primeiro método, graus mais baixos de fluorose medidos pelo índice TF foram identificados como de aparência satisfatória, enquanto que no segundo método também houve a mesma concordância, no entanto, os dentistas encontraram mais defeitos que os leigos, significando que os valores estéticos dos dentistas podem não refletir aqueles do restante da população.

Lalumandier e Rozier (1998) encontraram, utilizando o índice TSIF em 709 pacientes de 5 a 19 anos, 78\% com fluorose. Em relação à satisfação pessoal e dos pais quanto à cor dos dentes, $43 \%$ disseram estarem insatisfeitos, contudo, apenas os escores mais elevados do índice foram considerados essenciais para isso, justificando uma possível intervenção estética. Desta forma, os autores consideraram a fluorose como sendo ainda apenas um problema estético e não um problema em saúde pública, com a ressalva, no entanto, de que é necessário atenção por parte dos profissionais no uso adequado do flúor, preservando seu poder preventivo contra a cárie dentária e minimizando ainda mais o risco de instalação da fluorose. 
Furtado et al. (1999) encontraram em crianças de 6 a 12 anos de idade de Capão Alto, SC, 14,8\% com sinais clínicos da fluorose mensurado por meio do índice de Dean, sendo que 10,6\% dos casos eram questionáveis, em $0,8 \%$ dos casos houve fluorose leve, $1,5 \%$ fluorose moderada e em 1,9\% fluorose severa. Portanto, a fluorose dentária, para essa comunidade, não constituiu um problema de saúde pública.

Marcelino et al. (1999) publicaram os dados referentes a levantamento epidemiológico realizado na cidade de Araçatuba, cidade do interior paulista (com flúor agregado à água de abastecimento desde 1972, em concentração média de $0,7 \mathrm{mg} / \mathrm{L}$ ), obtidos a partir do exame de 317 crianças de 12 a 14 anos, utilizando o índice de Dean. Constataram que 24\% dessas apresentavam fluorose dentária em seus diversos graus e que o Índice de Fluorose da Comunidade (IFC) registrou um valor de 0,29 , não podendo ser considerada ainda a fluorose como um problema de saúde pública.

\section{Conclusões}

A fluorose dentária, apesar de poder ser considerada como um problema de saúde pública (STRIFFLER, 1956), será dependente direta e de modo especial do grau de severidade em que esteja acometendo uma determinada comunidade para que efetivamente passe a ser percebida pela população, trazendo consigo preocupação em termos de estética (LALUMANDIER e ROZIER, 1998).

Contrariamente à cárie dentária, que é uma doença multifatorial (NEWBRUN, 1978), a fluorose é uma alteração do esmalte dentário ocorrida durante o período de mineralização dos dentes (LESAN, 1987; BESTEN, 1994) sendo o risco de sua manifestação relacionado à exposição e ingestão, exclusivamente, de flúor em excesso, principalmente por meio dos dentifrícios, em crianças de baixa idade (STOOKEY, 1994; WANG et al., 1997; ARMONIA et al., 1999). Assim sendo, o controle e supervisão da higienização bucal das crianças por pais e educadores, de modo que a criança utilize pequena quantidade de creme dental, possibilitando a higienização oral e diminuindo a possibilidade de ingestão do dentifrício pela eliminação da maior parte da espuma formada, possivelmente diminuiria o risco de instalação das manifestações clínicas da fluorose. Além disso, em comunidades em que a água de abastecimento apresente teores 
naturais de flúor acima dos valores considerados terapêuticos no controle do processo de des-remineralização do esmalte dental, ações governamentais deveriam ser implementadas no sentido de se adequar essa condição para níveis considerados ótimos.

Recebido para publicação em 02/10/00.

Aceito para publicação em 12/12/00.

\begin{abstract}
Although dental decay is still considered the main problem of the mouth in Brazil, the decline in its prevalence and severity makes dentistry turn its attention to other problems, such as mouth cancer, malocclusion and dental fluorosis. An increase in the occurrence of dental fluorosis in the world and also in several areas of our country raises the question - Is it a public health problem? In an attempt to answer this question the authors analyse dental fluorosis and the characteristics that determine a problem in public health.
\end{abstract}

Key words: dental fluorosis; public health; fluorine

Endereço para contato: Vitoldo A. Kozlowski Junior

Universidade Estadual de Ponta Grossa

Praça Santos Andrade, n. 1

84010-919 - Ponta Grossa - PR

vakozlowski@uepg.br

\title{
REFERÊNCIAS
}

1 AKPATA, E. S.; FAKIHA, Z.; KHAN, N. Dental fluorosis in 12-15-year-old rural children exposed to fluorides from well drinking water in the Hail region of Saudi Arabia. Community Dent. Oral, Copenhagen, v. 25, n. 4, p. 324-327, Aug. 1997.

2 ARMONIA, P. L.; ADDE, C. A.; TORTAMANO, N.; BARROS, C. C. A.; SIMONE, J. L. Crema dental fluorada y fluorosis dental. Rev. Fol. Oral, São Paulo, v. 5, n. 15, p. 37-40, Mar./Jun. 1999. 
3 AZCURRA, A. I.; BATTELLINO, L. J.; CALAMARI, S. E.; CATTONI, S. T. D.; KREMER, M.; LAMBERGHINI, F. C. Estado de salud bucodental de escolares residentes en localidades abastecidas com agua de consumo humano de muy alto y muy bajo contenido de fluoruros. Rev. Saúde Pública, São Paulo, v. 29, n. 5 , p. 364-375, Out. 1995.

4 BARDSEN, A.; KLOCK, K. S.; BJORVATN, K. Dental fluorosis among persons exposed to high-and low-fluoride drinking water in western Norway. Community Dent. Oral, Copenhagen, v. 27, n. 4, p. 259-267, Aug. 1999.

5 BASTING, R. T.; PEREIRA, A. C.; MENEGHIM, M. C. Avaliação da prevalência de cárie dentária em escolares do município de Piracicaba, SP, Brasil, após 25 anos de fluoretação das águas de abastecimento público. Rev. Odontol. Univ. São Paulo, São Paulo, v. 11, n. 4, p. 287-292, out./dez. 1997.

6 BESTEN, P. K. Dental fluorosis: its use as biomarker. Adv. Dent. Res., Washington, v. 8, n. 1, p. 105-110, Jun. 1994.

7 BRUNELLE, J. A.; CARLOS, J. P. Recent trends in dental caries in US children and the effect of water fluoridation. J. Dent. Res., Washington, v. 69, p. 723-727, 1990. [Special Issue]

8 BUENDIA, O. C.; ZAINA, I. Será o flúor o vilão? Rev. Paul. Odontol., São Paulo, v. 19, n. 2, p. 36-45, mar./abr. 1997.

9 CARLOS, J. P. Fluoride mouth rinses. In: WEY, S. Clinical uses of fluoride. Philadelphia : Lea \& Febiger, cap. 6, p. 75-82, 1985.

10 CHAVES, M. M. Odontologia social. 3. ed. São Paulo : Artes Médicas, 1986. 448p.

11 CLARK, D. C.; HANN, H. J.; WILLIAMSON, M. F.; BERKOWITZ, J. Aesthetic concerns of children and parents in relation to different classifications of the tooth surface index of fuorosis. Community Dent. Oral, v. 21, p. 360-364. 1993.

12 CUNHA, J. P. P.; CUNHA, R. E. Sistema único de saúde: princípios. In: CAMPOS, F. E.; OLIVEIRA JR., M.; TONON, L. M. Cadernos de saúde 1. Belo Horizonte : Coopmed, 1998. p. 11-26.

13 DEAN, H. T. The investigation of physiological effects by the epidemiological method. In: MOULTON, F. R. Fluoride and dental health, Washington, p. 23-31, 1942. 
14 DOWNER, M. C. Impacto das mudanças de padrão de cárie dental. In: BOWEN, W. H.; TABAK, L. A. Cariologia para a década de 90. São Paulo : Santos, 1995. p. 25-35.

15 ELLWOOD, R. P.; O’MULLANE, D. Enamel opacities and dental esthetics. J. Public Health Dent., v. 55, n. 3, p. 171-176. 1995.

16 FEJERSKOV, O.; MANJI, F. Risk assessment in dental caries. In: BADER, J. D. et al. Risk assessment in dentistry. Chapel Hill : University of North Carolina Dental Ecology, 1990. p. 215-217.

17 FEJERSKOV, O.; MANJI, F.; BAELUM, V. The nature and mechanisms of dental fluorosis in man. J. Dent. Res., Washington, v. 69, Special Issue, p. 692700, 1990.

18 FEJERSKOV, O.; MANJI, F. Risk assessment in dental caries. In: BADER, J.D. Risk assessment in dentistry. Chapel Hill : Universty of North Carolina Dental Ecology, 1990. p. 215-217.

19 FORATTINI, O. P. Epidemiologia geral. 2. ed. São Paulo : Artes Médicas, 1996. 210p.

20 FREIRE, M. C. M.; PEREIRA, M. F.; BATISTA, S. M. O.; BORGES, M. R. S.; BARBOSA, M. I.; ROSA, A. G. F. Prevalência de cárie e necessidades de tratamento em escolares de seis a doze anos de idade, Goiânia, GO, Brasil, 1994. Rev. Saúde Pública, São Paulo, v. 31, n. 1, p. 44-52, fev. 1997.

21 FURTADO, A.; TRAEBERT, J. L.; MARCENES, W. S. Prevalência de doenças bucais e necessidade de tratamento em Capão Alto, Santa Catarina. Rev. ABO Nac., São Paulo, v. 7, n. 4, p. 226-230, ago./set. 1999.

22 HEIFETZ, S. B. Prevalence of dental caries and dental fluorosis in areas with optimal and above optimal water fluoride concentrations: a 5 year-follow-up survey. J. Am. Dent. Assoc., Chicago, v. 116, n. 4, p. 490-495, Apr. 1988.

23 HOROWITZ, H. S.; DRISCOLL, W. S.; MEYERS, R. J.; HEIFETZ, S. B.; KINGMAN, A. A new method for assessing the prevalence of dental fluorosis: the Tooth Surface Index of Fluorosis. J. Am. Dent. Assoc., Chicago, v. 109, n. 1, p. 37 41, 1984.

24 ISHI, T.; SUCKLING, G. The severity of dental fluorosis in children exposed to water a high fluoride for various periods of time. J. Dent. Res., Washington, v. 
70, n. 6, p. 952-956, 1991.

25 KEYES, P. H. The infections and transmissible nature of experimental dental caries. Arch. Oral Biol., Oxford, v. 1, n. 4, p. 304-320, 1960.

26 KRIGER, L. Promoção de saúde bucal. 2 ed. São Paulo : Artes Médicas, 1999. $475 \mathrm{p}$.

27 KUMAR, J. V.; GREEN, E. L.; WALLACE, W.; CARNAHAN, T. Trends in dental fluorosis and dental caries prevalences in Newburgh and Kingston, N. Y. Am. J. Public Health, New York, v. 79, n. 5, p. 565-569, 1989.

28 LALUMANDIER, J. A.; ROZIER, R. G. Parent's satisfaction with children's tooth color: fluorosis as a contributing factor. J. Am. Dent. Assoc., Chicago, v. 129, n. 7, p. 1000-1005, Jul. 1998.

29 LARSEN, M. J.; KIRKEGAARD, E.; POULSEN, S.; FEJERSKOV, O. Enamel fluoride, dental fluorosis and dental caries among immigrants to and permanent residents of five Danish fluoride areas. Caries Res., Basel, v. 20, n. 4, p. 349-355, 1986.

30 LESAN, W. R. Dental fluorosis, a review of literature with comments on tropical characteristics. East Afr. Med. J., Nairobi, v. 64, n. 7, p. 493-498, Jul. 1987.

31 LEVY, S. M. Review of fluoride exposures and ingestion. Community Dent. Oral, Copenhagen, v. 22, n. 3, p. 173-180, 1994.

32 LEVY, S. M.; ROZIER, R. G. Use of systemic fluoride supplement by North Carolina dentists. J. Am. Dent. Assoc., Chicago, v. 114, n. 3, p. 347-350, 1987.

33 MARCELINO, G.; GUIMARÃES, M. B.; SILVA, P. R.; TERRERI, A. L. M.; GUIMARÃES, L. O. C.; SALIBA, N. A. Fluorose dentária em escolares da rede pública na cidade de Araçatuba. Rev. Inst. Cienc. Saúde, São Paulo, v. 17, n. 2, p. 89-92, jul./dez. 1999.

34 McGAVRAN, E. G. What is public health. In: The practice of dental public health. Michigan : School of Public Health, 1956. Cont. Ed. Series, n. 67, p. 5-12.

35 MOIMAZ, S. A.; SALIBA, N. A.; ARCIERI, R. M.; SALIBA, O.; SUNDEFELD, M. L. M. M. Redução na prevalência da cárie dentária, após dez anos de fluoretação da água de abastecimento público, no município de Birigui, SP, Brasil. Rev. Faculdade Odontol. Lins, Lins, v. 8, n. 2, p. 41-45, jul./dez. 1995.

PUBLICATIO UEPG - Biological and Health Sciences, 6 (1): 75-87, 2000. 
36 MOLLER, I. J. Fluorides and dental fluorosis. Int. Dent. J., London, v. 32, n. 2, p. 135-147, Jun. 1982.

37 MURRAY, J. J. O uso correto de fluoretos na saúde pública. São Paulo : Santos, 1992. 131p.

38 NARVAI, P. C.; FRAZÃO, P.; CASTELLANOS, R. A. Declínio na experiência de cárie em dentes permanentes de escolares brasileiros no final do século XX. Odontol. Soc., São Paulo, v. 1, n. 1/2, p. 25-29, 1999.

39 NEWBRUN, E. Cariology. Baltimore : Williams \& Wilkins, 1978. 326 p.

40 ORGANIZAÇÃO Mundial de Saúde. Higiene dental. (Crônica de l. OMS), 9:11-6. 1954.

41 OSUJI, O. O.; LEAKE, J. L.; CHIPMAN, M. L.; NIKIFORUK, G.; LOCKER, D.; LEVINE, N. Risk factors for dental fluorosis in a fluoridated community. J. Dent. Res., v. 67, n. 12, p. 1488-1492. 1988.

42 PEREIRA, A. C.; CUNHA, F. L.; MENEGHIM, M. C.; WERNER, C. W. Dental caries and fluorosis prevalence study in a nonfluoridated Brazilian community: trend analysis and toothpaste association. J. Dent. Child., v. 67, n. 2, p. 132-135. 2000.

43 PEREIRA, A. C.; MOREIRA, B. H. W. Analysis of three dental fluorosis indexes used in epidemiologic trials. Brazilian Dent. J., Ribeirão Preto, v. 10, n. 1, p. 29-37, 1999

44 RIORDAN, P. J. Perceptions of dental fluorosis. J. Dent. Res., v. 72, n. 9, p. 1268-1274. 1993.

45 RIPA, L. W. A critique of topical fluoride methods (dentifrices, mouthrinses, operator, and self-applied gels) in an era of decreased caries and increased fluorosis prevalence. J. Public Health Dent., v. 51, n. 1, p. 23-41. 1991.

46 SEGRETO, V. A.; COLLINS, E. M.; CAMANN, D.; SMITH, C. T. A current study of mottled enamel in Texas. J. Am. Dent. Assoc., Chicago, v. 108, n. 1. p. 5659, 1984.

47 SHEIHAM, A. Changing trends in dental caries. Int. J. Epidemiol., Oxford, v. 13, p. 142-146, 1984. 
48 SINAI, N. Escola de Saúde Pública - Universidade de Michigan. In: CHAVES, M. M. Odontologia social. 3. ed. São Paulo : Artes Médicas, p. 24. 1986.

49 STEPHEN, K. W. Cárie nas populações jovens em todo o mundo. In: BOWEN, W. H.; TABAK, L. A. Cariologia para a década de 90. São Paulo : Santos, 1995. p. 37-50.

50 STOOKEY, G. K. Review of fluorosis risk of self-applied topical fluorides, dentifrices, mouthrinses and gels. Community Dent. Oral, Copenhagen, v. 22, n. 3, p. 181-186, 1994.

51 STRIFFLER, D. F. Planning a survey to secure a view of a state's oral health problem. In: The practice of dental public health. Michigan : School of Public Health, 1956. Cont. Ed. Series, n. 67, p. 126-127.

52 SZPUNAR, S. M.; BURT, B. A. Trends in the prevalence of dental fluorosis in the United States: a review. J. Public Health Dent., Richmond, v. 47, n. 2, p.71-79, 1987.

53 TAVARES, P. G.; BASTOS, J. R. M. Concentração de flúor na água: cárie, fluorose e teor de flúor urinário em escolares de Bauru - SP. Rev. Assoc. Paul. Cir. Dent., São Paulo, v. 53, n. 5, p. 407-415, set./out. 1999.

54 THYLSTRUP, A. Distribution of dental fluorosis in the primary dentition. Community Dent. Oral, Copenhagen, v. 6, n. 6, p. 329-337, Nov. 1978.

55 THYLSTRUP, A.; FEJERSKOV, O. Clinical appearance of dental fluorosis in permanent teeth in relation to histologic changes. Community Dent. Oral, Copenhagen, v. 6, n. 6, p. 315-328, Nov. 1978.

56 TRUIN, G. J.; KÖNIG, K. G.; BRONKHORST, E. M.; FRANKENMOLEN, F.; MULDER, J.; VAN'T HOF, M. A. Time trends in caries experience of 6 and 12year-old children of different socioeconomic status in The Hague. Caries Res., Basel, v. 32, n. 1, p. 1-4, Jan./Feb. 1998.

57 WANG, N. J.; GROPEN, A. M.; OGAARD, B. Risk factors associated with fluorosis in a non-fluoridated population in Norway. Community Dent. Oral, Copenhagen, v. 25, n. 6, p. 396-401, Dec. 1997. 